\title{
The effects of glycine on subjective daytime performance in partially sleep-restricted healthy volunteers
}

\author{
Makoto Bannai ${ }^{1}$, Nobuhiro Kawai ${ }^{1}$, Kaori Ono ${ }^{1}$, Keiko Nakahara $^{2}$ and Noboru Murakami ${ }^{2}$ \\ ${ }^{1}$ Frontier Research Labs, Institute for Innovation, Ajinomoto Co., Inc., Kanagawa, Japan \\ ${ }^{2}$ Veterinary Physiology, Faculty of Agriculture, University of Miyazaki, Miyazaki, Japan
}

\author{
Edited by: \\ Norio K. Ishida, National Institute of \\ Advanced Industrial Science and \\ Technology, Japan \\ Reviewed by: \\ Norio K. Ishida, National Institute of \\ Advanced Industrial Science and \\ Technology, Japan \\ Shaikh Mohammed lqbal, King Saud \\ University, Saudi Arabia \\ *Correspondence: \\ Makoto Bannai, Frontier Research \\ Lab, Institute for Innovation, \\ Ajinomoto Co., Inc., 1-1 Suzukicho, \\ Kawasaki, Kanagawa 210-8681, \\ Japan. \\ e-mail: makoto_bannai@ \\ ajinomoto.com
}

Approximately $30 \%$ of the general population suffers from insomnia. Given that insomnia causes many problems, amelioration of the symptoms is crucial. Recently, we found that a non-essential amino acid, glycine subjectively and objectively improves sleep quality in humans who have difficulty sleeping. We evaluated the effects of glycine on daytime sleepiness, fatigue, and performances in sleep-restricted healthy subjects. Sleep was restricted to $25 \%$ less than the usual sleep time for three consecutive nights. Before bedtime, $3 \mathrm{~g}$ of glycine or placebo were ingested, sleepiness, and fatigue were evaluated using the visual analog scale (VAS) and a questionnaire, and performance were estimated by personal computer (PC) performance test program on the following day. In subjects given glycine, the VAS data showed a significant reduction in fatigue and a tendency toward reduced sleepiness. These observations were also found via the questionnaire, indicating that glycine improves daytime sleepiness and fatigue induced by acute sleep restriction. PC performance test revealed significant improvement in psychomotor vigilance test. We also measured plasma melatonin and the expression of circadian-modulated genes expression in the rat suprachiasmatic nucleus (SCN) to evaluate the effects of glycine on circadian rhythms. Glycine did not show significant effects on plasma melatonin concentrations during either the dark or light period. Moreover, the expression levels of clock genes such as Bmal1 and Per2 remained unchanged. However, we observed a glycine-induced increase in the neuropeptides arginine vasopressin and vasoactive intestinal polypeptide in the light period. Although no alterations in the circadian clock itself were observed, our results indicate that glycine modulated SCN function. Thus, glycine modulates certain neuropeptides in the SCN and this phenomenon may indirectly contribute to improving the occasional sleepiness and fatigue induced by sleep restriction.

Keywords: amino acid, sleep restriction, circadian rhythm, sleepiness, fatigue

\section{INTRODUCTION}

Approximately $30 \%$ of the general population suffer from symptoms of insomnia (Ohayon, 2002). Insomnia-induced problems include cognitive inefficiency, sleepiness, mood disruptions (Zammit, 1988), impaired attention, and memory deficits (Hauri, 1997). Thus, treatment of insomnia is critical. To treat the symptoms, hypnotics are often used by those suffering from insomnia. Moreover, some natural herb extracts, such as chamomile (Shinomiya et al., 2005) and valerian (Fernandez-San-Martin et al., 2010) have been used to cure insomnia.

Recently, we found that glycine subjectively and objectively improved sleep quality in humans who suffered from repeated sleep complaints (Inagawa et al., 2006a; Yamadera et al., 2007). Glycine is a non-essential amino acid, and mammals can produce sufficient amounts of it via de novo synthesis. In humans, approximately $45 \mathrm{~g}$ of endogenous glycine is synthesized (Gersovitz et al., 1980), and 3-5 g of glycine is ingested from the daily diet. In both the peripheral and central nervous system, glycine acts as an inhibitory neurotransmitter via glycine receptors (Rajendra et al., 1997). Conversely, this amino acid also acts as an excitatory co-agonist via the $N$-methyl-D-aspartate subtype of glutamate receptors (NMDARs; Johnson and Ascher, 1987; Leeson and Iversen, 1994). Glycine has anti-inflammatory effects during ischemia, injury, and transplantation (Zhong et al., 2003). Moreover, glycine is known to attenuate the increase in fatty acids in rats fed on a high sucrose diet (El Hafidi et al., 2004).

In urban populations, most individuals experience restricted sleep. This is especially true for office workers in large cities, who are prone to restricted sleep periods during the week. During partial sleep restriction, daytime sleepiness and fatigue have been shown to increase, and daytime performance has been shown to be impaired (Dinges et al., 1997; Belenky et al., 2003; Van Dongen et al., 2003); these parameters can also be indicative of sleep quality. Taken together, improving sleep quality during sleep-restricted periods would ameliorate daytime sleepiness and fatigue. We investigated the use of glycine to improve sleep quality under partially sleep-restricted conditions using daytime sleepiness, fatigue, and performance as measurements.

To investigate the mechanisms by which glycine improves sleep, we assessed the permeability of the blood-brain barrier to glycine 
in a rat model (Kawai et al., 2012). The results indicated that ingested glycine passively diffuses across the blood-brain barrier by non-specific transportation. Furthermore, glycine improves sleep in rats by decreasing core body temperature and increasing vasodilatation (Kawai et al., 2009; Bannai and Kawai, 2011). The suprachiasmatic nucleus (SCN) is necessary for glycine-induced hypothermia and vasodilatation because ingested glycine primarily acts on the NMDARs in the SCN. This mechanism may result in an improvement in sleep quality. Although glycine has been shown to improve sleep quality in humans and rats (Inagawa et al., 2006a; Yamadera et al., 2007; Kawai et al., 2009; Bannai and Kawai, 2011), the relationship between glycine ingestion and the circadian clock remains unknown. The relationship between the clock gene and sleep are well known. For example, Per2 mutation results in advanced sleep phase syndrome (Toh et al., 2001; Xu et al., 2005), and Dec2 mutation causes short sleep phenotypes in humans (He et al., 2009). Melatonin is secreted by the pineal gland and circulates throughout the body. Melatonin concentrations are high during the dark period in all species, regardless of diurnal or nocturnal activity (Turek and Gillette, 2004; Arendt, 2005). Therefore, the timing of melatonin secretion is associated with circadian rhythms. The SCN, where many types of neuropeptides and clock genes are localized, is known as the circadian pacemaker site (Morin and Allen, 2006). We investigated the effects of glycine on melatonin concentrations in blood and the expression of neuropeptides and clock genes in the SCN.

\section{MATERIALS AND METHODS HUMAN STUDY \\ Participants}

The study protocol was approved by the Institutional Review Board of Ajinomoto Co., Inc. All of the participants enrolled in this study provided their written informed consent after receiving an explanation of the study procedure and the potential risks of the study. All of the procedures were carried out in accordance with Good Clinical Practice, the Helsinki Declaration, and related laws.

Ten healthy, male volunteers (30-61 years of age; average, 41.4 years) who were employed at Ajinomoto Co., Inc. as fulltime weekday workers participated. The subjects had good general health as determined by medical history and a medical examination. All of the participants were free of sleep disorders. To obtain background information on the sleep characteristics of the participants, the Pittsburgh Sleep Quality Index (PSQI) was used to evaluate their sleep patterns over the previous month (Buysse et al., 1989; Smyth, 1999; Doi et al., 2000). Furthermore, before the test period, the subjects were required to record a sleep log and fill out the visual analog scale (VAS) upon waking for 5 weekdays for the purposes of adaptation and acquiring baseline data. Substances such as alcohol, vitamin B12, melatonin, and other medication that may affect natural sleep were prohibited, and the participants' lifestyle patterns (except sleep) were kept constant during the test period. Among the 10 participants, 1 dropped out, and another 2 who took medication for influenza and a pollen allergy during the experimental period were excluded. Statistical analysis was performed on seven subjects (average, 40.6 years; PSQI score, $4.7 \pm 0.47)$.

\section{Methods}

This was a randomized single-blinded crossover trial. During the adaptation period (the first three consecutive nights), the participants spent the usual time in bed (TIB; average, $7.3 \mathrm{~h}$ ). During the experimental period, each participant took flavored glycine $(3 \mathrm{~g})$ or flavored placebo (a reduced form of malt sugar with the same flavor as glycine) $30 \mathrm{~min}$ before bedtime and their TIB was restricted to $25 \%$ less than usual (average, $5.5 \mathrm{~h}$ ) for three consecutive nights. Daytime naps were not permitted, and sleep was limited by delaying going to bed. Sleep duration was monitored by sleep logs.

On the first and last days of the experimental period (days 1 and 3 ) at 10:00, 14:00, and 18:00, the participants rated their own daytime sleepiness, fatigue, and performance using the VAS. In addition to the VAS, subjective daytime sleepiness and fatigue was recorded using a questionnaire for fatigue complaints (Jikakusho Shirabe) established by Japan Society for Occupational Health (Sasaki and Matsumoto, 2005). This questionnaire consisted of 25 items that included five factors (Factors I-V) drawn from the factor analysis and 5 subordinate items for each of those five factors. These five factors were as follows: feeling of drowsiness (Factor I), feeling of instability (Factor II), feeling of uneasiness (Factor III), feeling of local pain or dullness (Factor IV), and feeling of eyestrain (Factor V). Responses were measured on a five point rating scale ranging from "disagree completely" (1 point) to "agree strongly" (5 points). Performance was estimated by a sequence of the commercial test program "Performance Test Program" (NoruPro Light Systems, Inc., Tokyo, Japan). Test battery was composed of four different performance tasks and an instruction for each session was shown on the monitor at the beginning of the session. The first task was 3-min simple reaction test, in which participants were required to click as soon as possible when a symbol was displayed on the screen with a randomized interval (2-3 s). The second task was 4-min psychomotor vigilance test, where one of three symbols was randomly presented with a randomized interval (1.8-3 s), and the examinee was required to respond if the symbol was same as the target one. The third task was 3-min memory recognition task, where the test stimuli (a digit or an alphabet) was presented with an interval of $1.5 \mathrm{~s}$, and the examinee had to select right-click or left-click according to whether the stimuli was the same as the target (a certain digit or alphabet). The last task was number addition test, in which examinee was required to input the sum of two numbers of two digits.

After 7-14 recovery nights, the participants underwent another sequence of trials. During this time, the participants were given the flavored test substance that had not been administered previously.

\section{Statistical analysis}

Data are expressed as the mean \pm SE. A two-way analysis of variance (ANOVA) was used to evaluate the effects of glycine. Post hoc analyses were conducted using the Holm-Sidak method when significant differences $(p<0.05)$ were observed.

\section{RAT STUDY \\ Animals}

The rat experimental protocol was reviewed and approved by the Animal Care Committee of Ajinomoto. Male Wister rats 
(Charles River Japan, Yokohama, Japan) were purchased and housed individually in cages made of stainless steel wire. Rodent chow (CRF-1, Oriental Yeast, Tokyo, Japan) and tap water were available without restriction. The animal room was maintained at a constant temperature $\left(23 \pm 2^{\circ} \mathrm{C}\right)$ and humidity $(55 \pm 10 \%)$, with a 12-h light/dark cycle (lights on 7.00-19.00).

At 9.00 (light period) or 21.00 (dark period), $2 \mathrm{~g} / \mathrm{kg}$ glycine or vehicle (distilled water) was orally administered via gavage. The animals were anesthetized and sacrificed to collect blood and the brain $2 \mathrm{~h}$ later. The SCN was punched out from brain tissues for analysis.

\section{Melatonin assay and quantification of mRNA expression}

The melatonin concentrations present in plasma samples were determined by radioimmunoassay as described previously (Nakahara et al., 2002). The radioiodinated and non-radioactive melatonin used as standards were purchased from New England Nuclear and Sigma, respectively. The rabbit anti-melatonin serum (HAC-AA92-03RBP86) was supplied by Dr. Wakabayashi (Gunma University, Japan).

To quantify the mRNA expression of arginine vasopressin (AVP) and vasoactive intestinal polypeptide (VIP), total RNA was isolated from the SCN using the RNeasy Micro Kit (QIAGEN) and used for reverse transcription reactions with SuperScript ${ }^{\mathrm{TM}} \mathrm{II}$ RNase H Reverse Transcriptase (Invitrogen) according to the manufacturer's instructions. Quantitative PCR was performed using an ABI PRISM 7900HT (Applied Biosystems) with SYBR green fluorescence detection. Relative expression levels were normalized

to the $18 \mathrm{~S}$ expression levels in the same sample. The following primer sequences were used to detect the mRNA of each clock gene or neuropeptide:

$18 \mathrm{~S}$

Bmal1

Per2

AVP

VIP
Forward: CGCGGTTCTATTTTGTTGGTTTTCG Reverse: CTGATCGTCTTCGAACCTCCGACTT Forward: AACCCGTGGACCAAGGAAGT Reverse: GTGAGCTGTGGGAAGGTTGG Forward: CATGGCCAGTGTGCAGAGAG Reverse: GAGGCCACCAAACAGACAGG Forward: TCGCCATGATGCTCAACACT Reverse: GCCTCCTCTTGGGCAGTTCT Forward: GTTCCTGGCGATCCTGACAC Reverse: AGGCCAGCGACTGTGAGAAC

\section{Statistical analysis}

The data are expressed as the mean \pm SE. A two-way ANOVA was used to evaluate the effects of glycine. When a significant difference $(p<0.05)$ was observed, post hoc analyses were conducted using the Holm-Sidak method.

\section{RESULTS \\ HUMAN STUDY \\ Sleepiness and fatigue}

Data from the VAS test showed significant effects on fatigue on day $1(p=0.022)$; however, no significant effects were observed

Table 1 | Results and statistics for VAS and the questionnaire for fatigue complaints on day 1.

\begin{tabular}{|c|c|c|c|c|c|c|c|c|c|c|}
\hline & \multicolumn{6}{|c|}{ Results } & \multicolumn{4}{|c|}{ Statistics } \\
\hline & \multicolumn{3}{|c|}{ Placebo } & \multicolumn{3}{|c|}{ Glycine } & \multicolumn{2}{|c|}{ Treatment } & \multicolumn{2}{|c|}{ Treatment $\times$ time } \\
\hline & $10: 00$ & $14: 00$ & 18:00 & $10: 00$ & $14: 00$ & $18: 00$ & $F_{1,6}$ & $p$ & $F_{2,12}$ & $p$ \\
\hline \multicolumn{11}{|l|}{ VAS } \\
\hline $\begin{array}{l}\text { Sleepiness; Sleepy: } \\
100 \text { - alert: } 0\end{array}$ & $48.1(5.65)$ & $52.9(10.51)$ & $51(6.97)$ & $32.7(7.94)$ & $36(6.78)$ & $45.6(8.71)$ & 3.82 & 0.098 & 0.26 & 0.774 \\
\hline $\begin{array}{l}\text { Fatigue; Extremely } \\
\text { fatigued: } \\
100 \text { - vigorous: } 0\end{array}$ & $48.3(4.97)$ & $53.1(6.2)$ & $59.6(8.14)$ & $33.6(4.6)$ & $35.4(8.62)$ & $51(9.73)$ & 9.36 & 0.022 & 0.48 & 0.628 \\
\hline \multicolumn{11}{|c|}{ QUESTIONNAIRE FOR FATIGUE COMPLAINTS } \\
\hline $\begin{array}{l}\text { Factor } \\
\text { I-drowsiness }\end{array}$ & $11.7(1.46)$ & $11.7(1.55)$ & $10.1(1.79)$ & $8(0.79)$ & $7.4(0.84)$ & $8.9(0.99)$ & 5.03 & 0.066 & 0.38 & 0.691 \\
\hline Factor II - instability & $9.3(0.84)$ & $9.6(1.32)$ & $8(1.13)$ & $6.6(0.43)$ & $7(1)$ & $7.6(1.32)$ & 4.77 & 0.072 & 3.35 & 0.07 \\
\hline $\begin{array}{l}\text { Factor } \\
\text { III - uneasiness }\end{array}$ & $9(0.98)$ & $8.9(1.18)$ & $9(2.36)$ & $7.6(0.43)$ & $7.4(0.81)$ & $8.4(1.02)$ & 1.55 & 0.259 & 0.25 & 0.78 \\
\hline $\begin{array}{l}\text { Factor IV - local pain } \\
\text { or dullness }\end{array}$ & $7.7(0.64)$ & $7.4(0.53)$ & $7.1(0.74)$ & $6.6(0.57)$ & $6.4(0.53)$ & $6.7(0.47)$ & 3.16 & 0.125 & 0.51 & 0.611 \\
\hline Factor $\mathrm{V}$ - eyestrains & $9.4(1.51)$ & $8.9(1.53)$ & $9.6(1.17)$ & $7.4(1.09)$ & $8(1.18)$ & $8.4(1.43)$ & 4.41 & 0.081 & 0.61 & 0.559 \\
\hline $\begin{array}{l}\text { Total score; } \\
\text { extremely fatigued: } \\
125 \text { - vigorous: } 25\end{array}$ & $47(4.24)$ & $46.1(4.93)$ & $43.7(6.17)$ & $36.1(1.75)$ & $36.3(3.21)$ & $40(4.21)$ & 4.44 & 0.08 & 1.2 & 0.334 \\
\hline
\end{tabular}

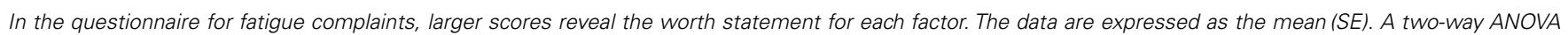
was used to test statistical significance. 
for sleepiness $(p=0.098$, Table 1$)$. Post hoc analysis revealed a significant improvement in fatigue at 14:00 $(p=0.022)$. Table 1 also shows the results of the questionnaire for fatigue complaints. No significant effects were observed between the placebo and glycine treatment groups for any measurement; however, some of the questions showed a tendency toward differences in drowsiness $(p=0.066)$, instability $(p=0.072)$, and eyestrain $(p=0.081)$ and total score $(p=0.080)$.
On day 3, no significant difference was observed between the placebo and glycine treatment groups.

\section{Performance test}

The results of the test on day 1 and day 3 were shown in Figures 1 and 2, respectively, and statistical analysis of the test on day 1 and day 3 were shown in Table 2 . In the psychomotor vigilance test, reaction time was significantly decreased in glycine group on both

\section{Simple reaction test}

A

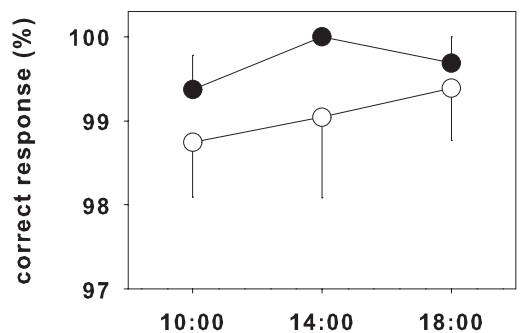

Psychomotor vigilance test

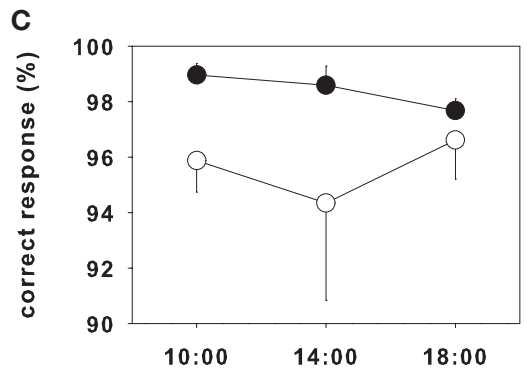

Memory recognition test

E

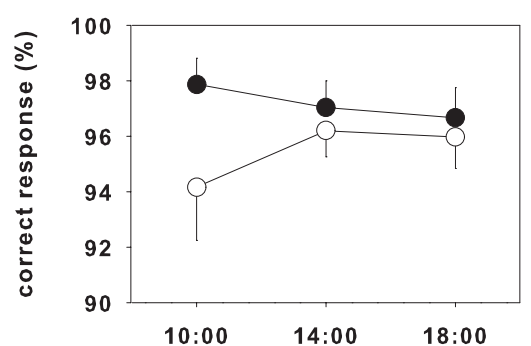

Number addition test

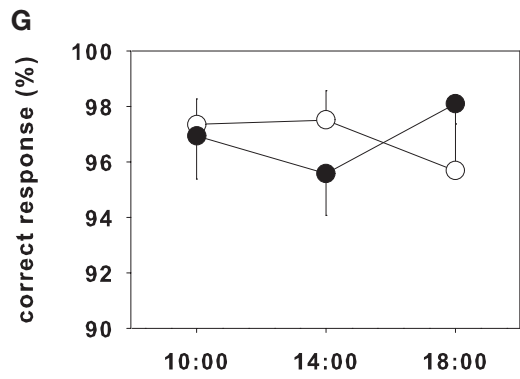

FIGURE 1 | Performance test on day 1. Closed and opened symbols reveal glycine and placebo ingested group, respectively. Asterisk shows significant differences between glycine and placebo groups. Simple reaction test $\mathbf{( A , B )}$

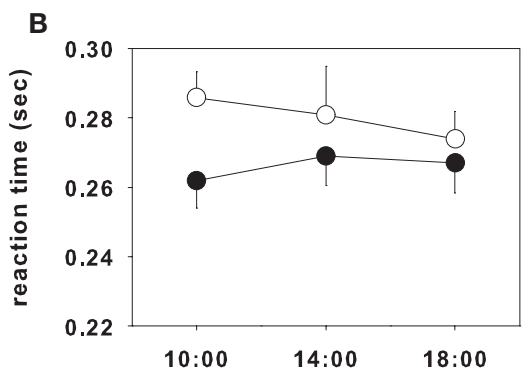

D

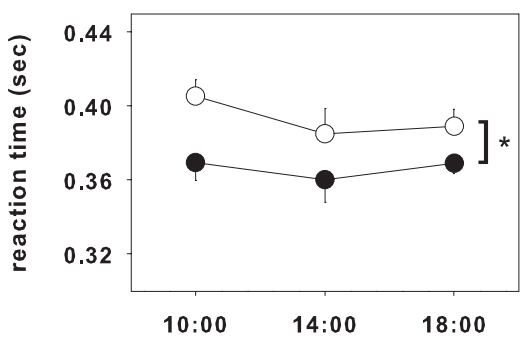

$\mathbf{F}$

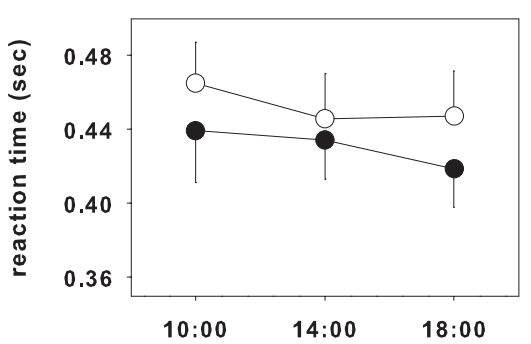

H

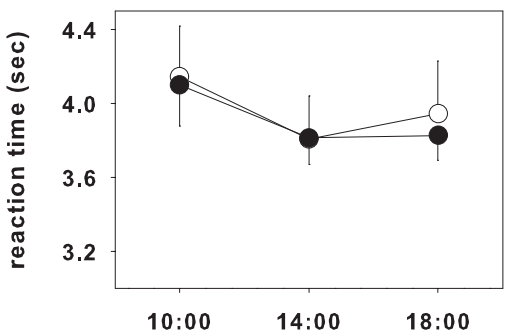

memory recognition test $(\mathbf{E}, \mathbf{F})$ and number addition test $\mathbf{( G , H )}$ showed no significance. Psychomotor vigilance test showed significant effects in reaction time (D), however that of correct response did not showed significance (C). 

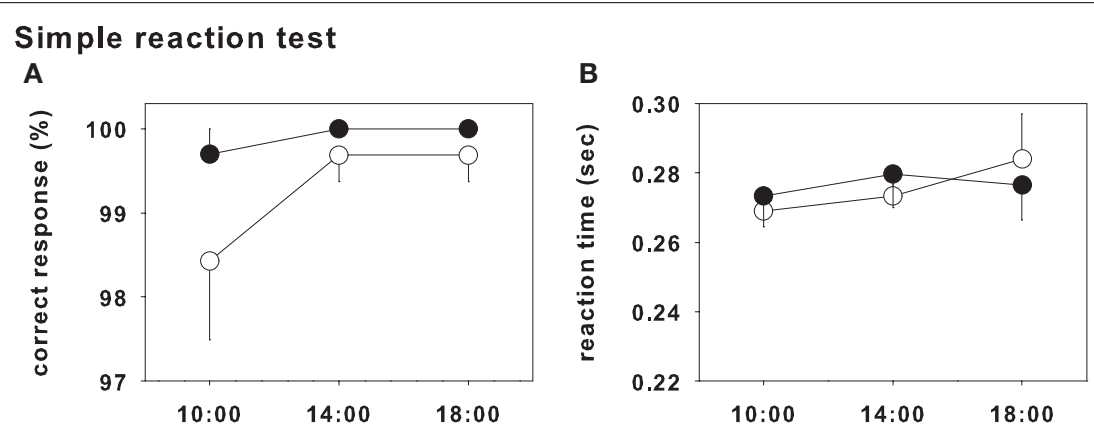

\section{Psychomotor vigilance test}
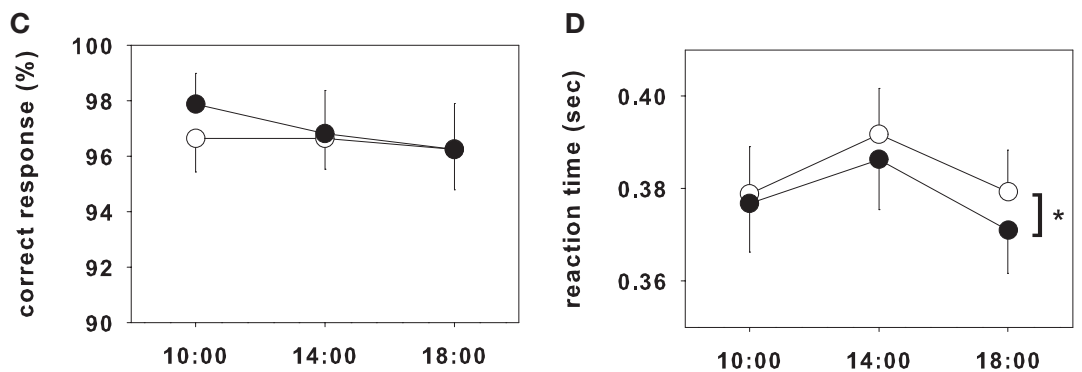

Memory recognition test
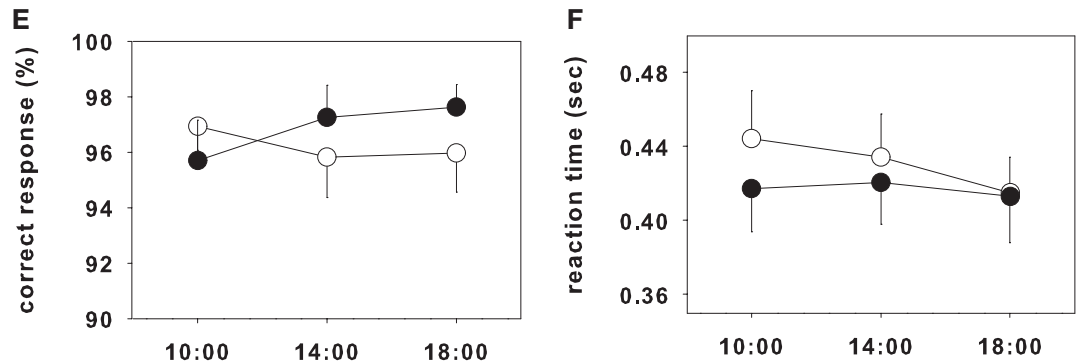

\section{Number addition test}

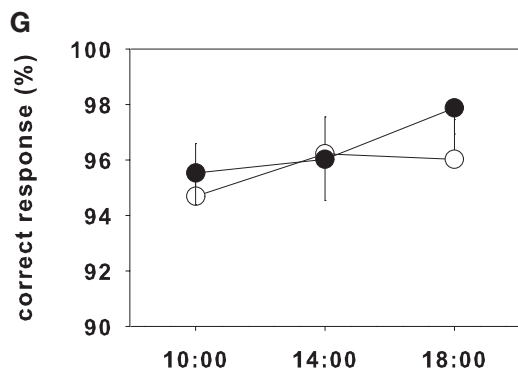

FIGURE 2 | Performance test on day 3. Closed and opened symbols reveal glycine and placebo ingested group, respectively. Asterisk shows significant differences between glycine and placebo groups. Simple reaction test $(\mathbf{A}, \mathbf{B})$,

day 1 and day 3 (Figures 1D and 2D). While the other tests did not show significant differences, glycine treatment group showed a tendency toward improvement.

\section{RAT STUDY}

\section{Melatonin assay}

Plasma melatonin concentrations were not significantly different between the glycine and vehicle treatment groups (Figure 3).
H

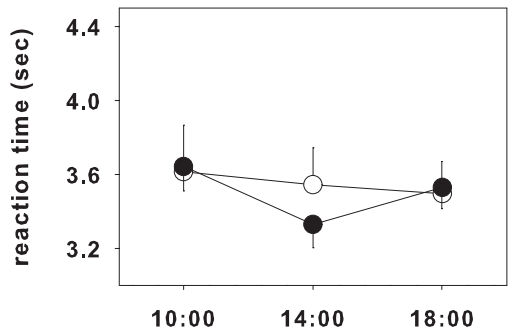

memory recognition test $(\mathbf{E}, \mathbf{F})$ and number addition test $\mathbf{( G , H )}$ showed no significance. Psychomotor vigilance test showed significant effects in reaction time (D), however that of correct response did not showed significance (C).

\section{Quantification of mRNA expression}

The expression of the clock genes Bmall and Per2 and the neuropeptides AVP and VIP in the SCN were measured following glycine or vehicle administration. Although Bmal1 and Per1 mRNA expression did not show significant differences between the glycine and vehicle treatment groups (data not shown), AVP and VIP were significantly increased during the light period in the glycine group (Figures 4A,B). 


\begin{tabular}{|c|c|c|c|c|c|c|c|c|}
\hline & \multicolumn{4}{|c|}{ Day 1} & \multicolumn{4}{|c|}{ Day 3} \\
\hline & \multicolumn{2}{|c|}{ Treatment } & \multicolumn{2}{|c|}{ Treatment $x$ time } & \multicolumn{2}{|c|}{ Treatment } & \multicolumn{2}{|c|}{ Treatment $\times$ time } \\
\hline & $F_{1,6}$ & $p$ & $F_{2,12}$ & $p$ & $F_{1,6}$ & $\boldsymbol{p}$ & $F_{2,12}$ & $\boldsymbol{p}$ \\
\hline \multicolumn{9}{|c|}{ SIMPLE REACTION TIMETEST } \\
\hline Correct response & 1.35 & 0.289 & 0.18 & 0.838 & 1.36 & 0.288 & 1.4 & 0.284 \\
\hline Reaction time & 3.78 & 0.1 & 1.78 & 0.211 & 0.02 & 0.892 & 1.09 & 0.369 \\
\hline \multicolumn{9}{|c|}{ PSYCHOMOTOR VIGILANCE TEST } \\
\hline Correct response & 2.84 & 0.143 & 1.41 & 0.283 & 0.4 & 0.55 & 0.71 & 0.513 \\
\hline Reaction time & 21.69 & 0.003 & 0.66 & 0.536 & 6.01 & 0.05 & 0.07 & 0.937 \\
\hline \multicolumn{9}{|c|}{ MEMORY RECOGNITIONTEST } \\
\hline Correct response & 4.57 & 0.076 & 2.65 & 0.111 & 1.54 & 0.261 & 4.08 & 0.044 \\
\hline Reaction time & 1.7 & 0.24 & 1 & 0.397 & 0.73 & 0.426 & 0.53 & 0.602 \\
\hline \multicolumn{9}{|c|}{ NUMBER ADDITION TEST } \\
\hline Correct response & 0.0004 & 0.984 & 2.1 & 0.165 & 0.55 & 0.486 & 0.89 & 0.435 \\
\hline Reaction time & 0.05 & 0.838 & 0.19 & 0.831 & 0.07 & 0.795 & 1.32 & 0.304 \\
\hline
\end{tabular}

The data are expressed as the mean \pm SE. A two-way ANOVA was used to test statistical significance.

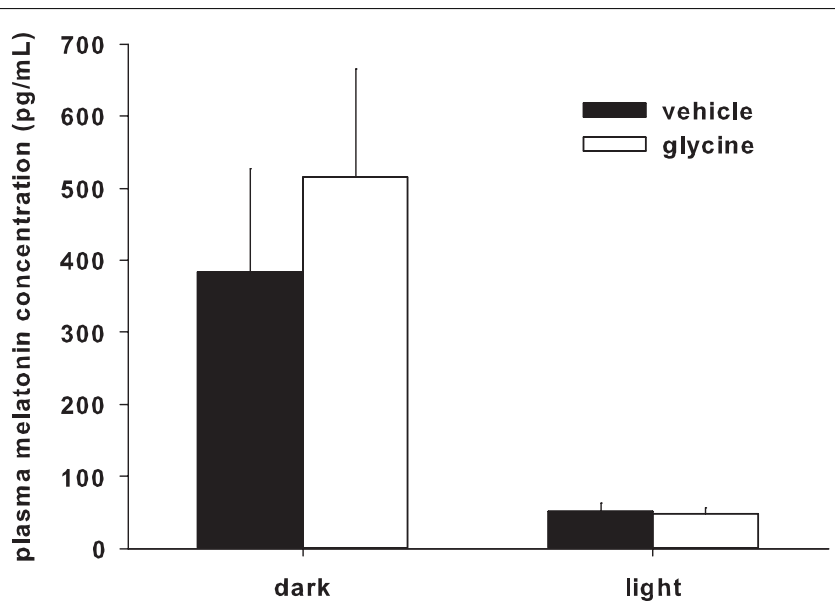

FIGURE 3 | Plasma melatonin concentration. Plasma melatonin concentration was not changed by glycine administration.

\section{DISCUSSION}

Most individuals experience periods of sleep deprivation. In the first half of this study, the ingestion of $3 \mathrm{~g}$ of glycine before bedtime ameliorated daytime fatigue under modest sleep restriction conditions. These improvements were observed on the first day but not on the third day of three consecutive sleep-restricted days. Additionally, performance which was evaluated by psychomotor vigilance test was also improved on both first and third days. Thus, glycine improved subjective parameters, such as fatigue, only on day 1; however, objective parameters, such as psychomotor vigilance, were improved on both days 1 and 3. The reason for the continued improvement in psychomotor vigilance until day 3 is not clear, and further study is needed on the improvement of both subjective and objective parameters by glycine ingestion. Our results indicate that the ingestion of glycine at bedtime occasionally improves the impairments in subjective alertness and neurobehavioral functions induced by acute and modest sleep restriction.

Previously, we showed the effects of glycine on sleep quality in humans who had continuously complained about their sleep quality (Inagawa et al., 2006a,b; Yamadera et al., 2007). In these studies, ingestion of $3 \mathrm{~g}$ glycine was found to improve subjective sleep quality (Inagawa et al., 2006a) and shorten sleep latency (Yamadera et al., 2007) as measured by polysomnography. In the current study, the participants had no sleep complaints, and the average of their PSQI scores was 4.7. These scores indicate that the participants had objectively normal sleep. Sleep was restricted to $25 \%$ less than usual for three consecutive days (i.e., the normal TIB for our participants was $7.3 \mathrm{~h}$, and the average TIB during the experiment was $5.5 \mathrm{~h}$ ). For most individuals, this decrease in sleep time occurs occasionally in normal life. Recent studies have indicated that consecutive days of partial sleep restriction could induce adverse effects on subjective sleepiness and neurobehavioral functions similar to those induced by total sleep deprivation (Belenky et al., 2003; Van Dongen et al., 2003; Vgontzas et al., 2004). In our experiment, we intended to induce these adverse effects in our subjects. Glycine significantly improved feelings of fatigue compared with the placebo and showed a tendency to reduce sleepiness (Table 1) and significant improvement in performance (Figures 1 and 2; Table 2). These results indicate that glycine improves sleep quality not only in people suffering from light insomnia but also in those with occasional sleep restrictions.

We previously described the pharmacokinetics of orally administered glycine in rats (Kawai et al., 2012). Gavage injection of glycine was found to increase both the plasma and cerebrospinal fluid levels of glycine and to passively diffuse across the blood-brain barrier via non-specific transportation. Furthermore, pharmacological findings showed that glycine primarily acts on NMDARs in the SCN and decreases the body core temperature via vasodilatation (Kawai et al., 2009; Bannai and Kawai, 2011). The relationship between sleep and core body temperature is very strong. Core body temperature has a circadian oscillation, i.e., it 


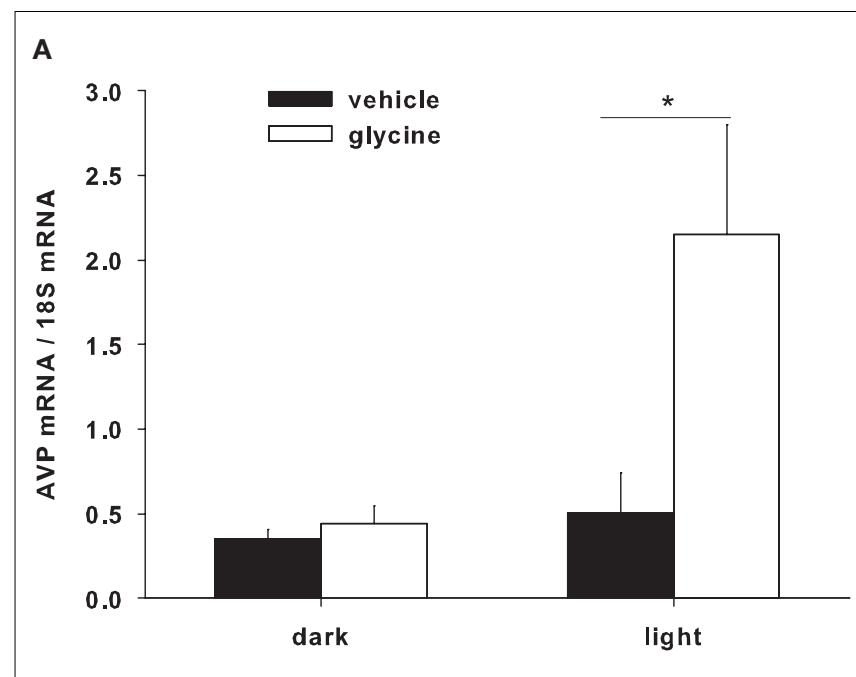

B

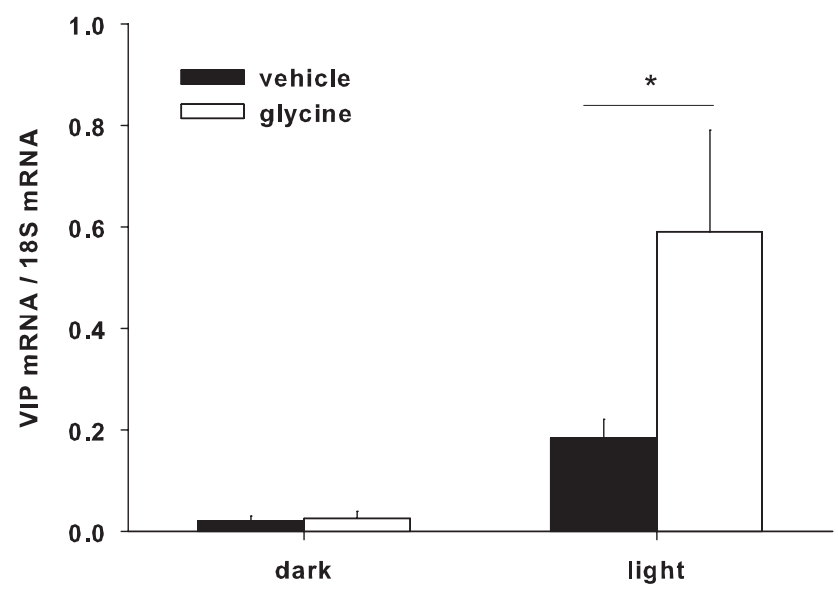

FIGURE 4 | Gene expression in the SCN. The expression of AVP (A) and VIP (B) were significantly increased during the light period after glycine administration.

drops at the onset of sleep (Czeisler et al., 1980; Zulley et al., 1981), continues to decrease during sleep (Barrett et al., 1993) and gradually rises as a person wakes (Foret et al., 1993). Thus, the heat dissipation induced by glycine ingestion leads to an improvement in sleep quality.

Although glycine improves sleep by reducing core body temperature, the effects of glycine on circadian rhythms are still unknown. Therefore, we investigated the effects of glycine on plasma melatonin concentration and the mRNA expression of

\section{REFERENCES}

Arendt, J. (2005). Melatonin: characteristics, concerns, and prospects. $J$. Biol. Rhythms 20, 291-303.

Ban, Y., Shigeyoshi, Y., and Okamura, H. (1997). Development of vasoactive intestinal peptide mRNA rhythm in the rat suprachiasmatic nucleus. J. Neurosci. 17, 3920-3931.
Bannai, M., and Kawai, N. (2011). New therapeutic strategy for amino acid medicine:glycine improves the quality of sleep. J. Pharmacol. Sci. 118, 145-148.

Barrett, J., Lack, L., and Morris, M. (1993). The sleep-evoked decrease of body temperature. Sleep 16, 93-99.

Belenky, G., Wesensten, N. J., Thorne, D. R., Thomas, M. L., Sing, H.

clock genes and neuropeptides in the SCN. Plasma melatonin concentration has been shown to be high during the nocturnal period, and ingestion of melatonin is known to induce sleep (Turek and Gillette, 2004; Arendt, 2005). If the plasma melatonin concentration is indeed increased by glycine ingestion, this phenomenon could be a possible mechanism for sleep improvement by glycine. We found, however, that the melatonin levels were not significantly changed by gavage glycine administration in either the diurnal and nocturnal period, suggesting that melatonin is not related to glycine administration. In addition, we measured the mRNA expression of known clock genes and neuropeptides in the SCN. Bmal1 and Per2 are the canonical clock genes expressed in the SCN (Sakamoto et al., 1998; Oishi et al., 2000; Fahrenkrug et al., 2008; Dzirbikova et al., 2011). The expression of these genes was not significantly changed by glycine administration, suggesting that glycine does not primarily affect the circadian rhythms. The expression levels of AVP and VIP in the SCN, however, were significantly increased during the light period (Figures 4A,B). Previously, the expression levels of these neuropeptides were shown to oscillate with the circadian rhythm, and expression was shown to be high at the end of the light period and decreased to low levels during light period (Ban et al., 1997; Yambe et al., 2002; Kalamatianos et al., 2004). Our results showed higher mRNA expression of both peptides during the light period in control rats; however, the differences between dark and light expression were very small in control group. For this experiment, we performed quantitative RT-PCR, and it is difficult to evaluate small differences in expression with this method. Glycine increased AVP and VIP expression 4.2- and 3.2-fold, respectively, during the light period. Thus, we believe that glycine has significant effects on the expression levels of these neuropeptides during the light period. Additionally, there were no significant effects of glycine on blood AVP concentrations, blood pressure, or heart rate (data not shown). The localization of each mRNA is different in the SCN. For instance, VIP is expressed in the ventrolateral component of the SCN (i.e., the core), AVP is expressed in the dorsomedial component of the SCN (i.e., the shell; Moore, 1993). In addition, AVP and VIP are known to interact each other in the SCN (Tamada et al., 1998). The SCN has an afferent projection to the medial preoptic area (mPOA; Deurveilher et al., 2002), an area known to be involved in thermoregulation (Kumar et al., 2007). Thus, glycine may modulate SCN function and neurotransmission to the $\mathrm{mPOA}$ via the regulation of AVP and VIP expression.

\section{ACKNOWLEDGMENTS}

This study was supported in part by the Program for Promotion of Basic Research Activities for Innovative Bioscience (PROBRAIN).

C., Redmond, D. P., Russo, M. B., and Balkin, T. J. (2003). Patterns of performance degradation and restoration during sleep restriction and subsequent recovery: a sleep dose-response study. J. Sleep Res. 12, 1-12.

Buysse, D. J., Reynolds, C. F. III, Monk, T. H., Berman, S. R., and Kupfer, D. J. (1989). The
Pittsburgh Sleep Quality Index: a new instrument for psychiatric practice and research. Psychiatry Res 28, 193-213.

Czeisler, C. A., Weitzman, E., MooreEde, M. C., Zimmerman, J. C., and Knauer, R. S. (1980). Human sleep: its duration and organization depend on its circadian phase. Science 210, 1264-1267. 
Deurveilher, S., Burns, J., and Semba, K. (2002). Indirect projections from the suprachiasmatic nucleus to the ventrolateral preoptic nucleus: a dual tract-tracing study in rat. Eur. $J$. Neurosci. 16, 1195-1213.

Dinges, D. F., Pack, F., Williams, K., Gillen, K. A., Powell, J. W., Ott, G. E., Aptowicz, C., and Pack, A. I. (1997). Cumulative sleepiness, mood disturbance, and psychomotor vigilance performance decrements during a week of sleep restricted to 4-5 hours per night. Sleep 20, 267-277.

Doi, Y., Minowa, M., Uchiyama, M., Okawa, M., Kim, K., Shibui, K., and Kamei, Y. (2000). Psychometric assessment of subjective sleep quality using the Japanese version of the Pittsburgh Sleep Quality Index (PSQI-J) in psychiatric disordered and control subjects. Psychiatry Res 97, 165-172.

Dzirbikova, Z., Kiss, A., Okuliarova, M., Kopkan, L., and Cervenka, L. (2011). Expressions of perl clock gene and genes of signaling peptides vasopressin, vasoactive intestinal peptide, and oxytocin in the suprachiasmatic and paraventricular nuclei of hypertensive TGR[mREN2]27 rats. Cell. Mol. Neurobiol. 31, 225-232.

El Hafidi, M., Perez, I., Zamora, J., Soto, V., Carvajal-Sandoval, G., and Banos, G. (2004). Glycine intake decreases plasma free fatty acids, adipose cell size, and blood pressure in sucrosefed rats. Am. J. Physiol. Regul. Integr. Comp. Physiol. 287, R1387-R1393.

Fahrenkrug, J., Hannibal, J., and Georg, B. (2008). Diurnal rhythmicity of the canonical clock genes Per1, Per2 and Bmall in the rat adrenal gland is unaltered after hypophysectomy. $J$. Neuroendocrinol. 20, 323-329.

Fernandez-San-Martin, M. I., MasaFont, R., Palacios-Soler, L., SanchoGomez, P., Calbo-Caldentey, C., and Flores-Mateo, G. (2010). Effectiveness of valerian on insomnia: a metaanalysis of randomized placebocontrolled trials. Sleep Med. 11, 505-511.

Foret, J., Aguirre, A., Touitou, Y., Clodore, M., and Benoit, O. (1993). Effect of morning bright light on body temperature, plasma cortisol and wrist motility measured during 24 hour of constant conditions. Neurosci. Lett. 155, 155-158.

Gersovitz, M., Bier, D., Matthews, D., Udall, J., Munro, H. N., and Young, V. R. (1980). Dynamic aspects of whole body glycine metabolism: influence of protein intake in young adult and elderly males. Metab. Clin. Exp. 29, 1087-1094.

Hauri, P. J. (1997). Cognitive deficits in insomnia patients. Acta Neurol. Belg. 97, 113-117.
He, Y., Jones, C. R., Fujiki, N., Xu, Y., Guo, B., Holder, J. L., Rossner, M. J., Nishino, S., and Fu, Y.-H. (2009). The transcriptional repressor DEC2 regulates sleep length in mammals. Science 325, 866-870.

Inagawa, K., Hiraoka, T., Kohda, T., Yamadera, W., and Takahashi, M. (2006a). Subjective effects of glycine ingestion before bedtime on sleep quality. Sleep Biol. Rhythms 4, 75-77.

Inagawa, K., Kawai, N., Ono, K. Sukegawa, E., Tsubuku, S., and Takahashi, M. (2006b). Assessment of acute adverse events of glycine ingestion at a high dose in human volunteers. Seikatsu Eisei 50, 27-32.

Johnson, J. W., and Ascher, P. (1987). Glycine potentiates the NMDA response in cultured mouse brain neurons. Nature 325, 529-531.

Kalamatianos, T., Kalló, I., and Coen, C. W. (2004). Ageing and the diurnal expression of the mRNAs for vasopressin and for the Vla and V1b vasopressin receptors in the suprachiasmatic nucleus of male rats. J. Neuroendocrinol. 16, 493-501.

Kawai, N., Bannai, M., Seki, S. Koizumi, T., Shinkai, K., Nagao, K., Matsuzawa, D., Takahashi, M., and Shimizu, E. (2012). Pharmacokinetics and cerebral distribution of glycine administered to rats. Amino acids. PMID: 21647662. [Epub ahead of print].

Kawai, N., Bannai, M., and Takahashi, M. (2009). "Glycine decreases core body temperature and increases cutaneous blood flow via NMDA receptors in rats," in Neuroscience 2009, Abstract.

Kumar, V. M., Vetrivelan, R., and Mallick, H. N. (2007). Noradrenergic afferents and receptors in the medial preoptic area: neuroanatomical and neurochemical links between the regulation of sleep and body temperature. Neurochem. Int. 50, 783-790.

Leeson, P. D., and Iversen, L. L. (1994), The glycine site on the NMDA receptor: structure-activity relationships and therapeutic potential. J. Med. Chem. 37, 4053-4067.

Moore, R. Y. (1993). Organization of the primate circadian system. J. Biol. Rhythms 8(Suppl.), S3-S9.

Morin, L. P., and Allen, C. N. (2006). The circadian visual system, 2005. Brain Res. Rev. 51, 1-60.

Nakahara, K., Abe, Y., Murakami, T., Shiota, K., and Murakami, N. (2002). Pituitary adenylate cyclaseactivating polypeptide (PACAP) is involved in melatonin release via the specific receptor PACAP-rl, but not in the circadian oscillator, in chick pineal cells. Brain Res. 939, 19-25.
Ohayon, M. M. (2002). Epidemiology of insomnia: what we know and what we still need to learn. Sleep Med. Rev 6, 97-111.

Oishi, K., Fukui, H., and Ishida, N. (2000). Rhythmic expression of BMAL1 mRNA is altered in clock mutant mice: differential regulation in the suprachiasmatic nucleus and peripheral tissues. Biochem. Biophys. Res. Commun. 268, 164-171.

Rajendra, S., Lynch, J. W., and Schofield, P. R. (1997). The glycine receptor. Pharmacol. Ther. 73, 121-146.

Sakamoto, K., Nagase, T., Fukui, H., Horikawa, K., Okada, T., Tanaka, H., Sato, K., Miyake, Y., Ohara, O., Kako, K., and Ishida, N. (1998). Multitissue circadian expression of rat periodhomolog (rPer2) mRNA is governed by the mammalian circadian clock, the suprachiasmatic nucleus in the brain. J. Biol. Chem. 273 , 27039-27042.

Sasaki, T., and Matsumoto, S. (2005). Actual conditions of work, fatigue and sleep in non-employed, homebased female information technology workers with preschool children. Ind. Health 43, 142-150.

Shinomiya, K., Inoue, T., Utsu, Y., Tokunaga, S., Masuoka, T., Ohmori, A. and Kamei, C. (2005). Hypnotic activities of chamomile and passiflora extracts in sleep-disturbed rats. Biol. Pharm. Bull. 28, 808-810.

Smyth, C. (1999). The Pittsburgh Sleep Quality Index (PSQI). J. Gerontol. Nurs. 25, 10-11.

Tamada, Y., Tanaka, M., Munekawa, K., Hayashi, S., Okamura, H. Kubo, T., Hisa, Y., and Ibata, Y. (1998). Neuron-glia interaction in the suprachiasmatic nucleus: a double labeling light and electron microscopic immunocytochemical study in the rat. Brain Res. Bull. 45, 281-287.

Toh, K. L., Jones, C. R., He, Y., Eide, E. J., Hinz, W. A., Virshup, D. M., Ptácek, L. J., and Fu, Y.-H. (2001). An hPer2 phosphorylation site mutation in familial advanced sleep phase syndrome. Science 291, 1040-1043.

Turek, F. W., and Gillette, M. U. (2004). Melatonin, sleep, and circadian rhythms: rationale for development of specific melatonin agonists. Sleep Med. 5, 523-532.

Van Dongen, H. P., Maislin, G., Mullington, J. M., and Dinges, D. F. (2003). The cumulative cost of additional wakefulness: dose-response effects on neurobehavioral functions and sleep physiology from chronic sleep restriction and total sleep deprivation. Sleep 26, 117-126.

Vgontzas, A. N., Zoumakis, E., Bixler, E. O., Lin, H. M., Follett, H., Kales, A., and Chrousos, G. P. (2004). Adverse effects of modest sleep restriction on sleepiness, performance, and inflammatory cytokines. J. Clin. Endocrinol. Metab. 89, 2119-2126.

Xu, Y., Padiath, Q. S., Shapiro, R. E., Jones, C. R., Wu, S. C., Saigoh, N., Saigoh, K., Ptacek, L. J., and Fu, Y.H. (2005). Functional consequences of a CKI[delta] mutation causing familial advanced sleep phase syndrome. Nature 434, 640-644.

Yamadera, W., Inagawa, K., Chiba, S., Bannai, M., Takahashi, M., and Nakayama, K. (2007). Glycine ingestion improves subjective sleep quality in human volunteers, correlating with polysomnographic changes. Sleep Biol. Rhythms 5, 126-131.

Yambe, Y., Arima, H., Kakiya, S., Murase, T., and Oiso, Y. (2002). Diurnal changes in arginine vasopressin gene transcription in the rat suprachiasmatic nucleus. Brain Res. Mol. Brain Res. 104, 132-136.

Zammit, G. K. (1988). Subjective ratings of the characteristics and sequelae of good and poor sleep in normals. $J$. Clin. Psychol. 44, 123-130.

Zhong, Z., Wheeler, M. D., Li, X., Froh, M., Schemmer, P., Yin, M., Bunzendaul, H., Bradford, B., and Lemasters, J. J. (2003). L-Glycine: a novel antiinflammatory, immunomodulatory, and cytoprotective agent. Curr. Opin. Clin. Nutr. Metab. Care 6, 229-240.

Zulley, J., Wever, R., and Aschoff, J. (1981). The dependence of onset and duration of sleep on the circadian rhythm of rectal temperature. Pflugers Arch. 391, 314-318

Conflict of Interest Statement: The authors declare that the research was conducted in the absence of any commercial or financial relationships that could be construed as a potential conflict of interest.

Received: 20 September 2011; accepted: 29 March 2012; published online: 18 April 2012.

Citation: Bannai M, Kawai N, Ono K, Nakahara K and Murakami N (2012) The effects of glycine on subjective daytime performance in partially sleeprestricted healthy volunteers. Front. Neur. 3:61. doi: 10.3389/fneur.2012.00061

This article was submitted to Frontiers in Sleep and Chronobiology, a specialty of Frontiers in Neurology.

Copyright $\odot 2012$ Bannai, Kawai, Ono, Nakahara and Murakami. This is an open-access article distributed under the terms of the Creative Commons Attribution Non Commercial License, which permits non-commercial use, distribution, and reproduction in other forums, provided the original authors and source are credited. 